\title{
SLJID reviewers 2019 (September) - 2020 (December)
}

The Editorial board of the SLID wishes to express their gratitude to the reviewers who immensely contributed to improve the quality of work published in the journal by reviewing manuscripts from September 2019 to December 2020. Their timely responses despite the ongoing COVID-19 pandemic, which has increased the workload of all involved in infectious diseases, is greatly appreciated.

\begin{tabular}{|c|c|c|}
\hline Amarasinghe Ananda & Jayasinghe Saroj & Samarasinghe Sagarika \\
\hline Ariyaratne Chamila & Jayathilake Kushlanie & Senanayake Channa \\
\hline Athauda-arachchi Pandula & Jayawardena Dushyanthie & Senanayake Nilanthi \\
\hline \multirow[t]{2}{*}{ Abeywardena Madhumanee } & & Senanayake Sanath \\
\hline & Karunaratne Nedra & Sigera Samithra \\
\hline \multirow[t]{2}{*}{ Badanasinghe Nadisha } & Karunathilake Thilina & Siriwardana Yamuna \\
\hline & Kalupahana Ruwani & Sumathipala Gayan \\
\hline Chandrasiri Shirani & Ketheesan Natkunam & Suranadee Sumudu \\
\hline Chinniah TR & Kothalawala Mahen & Sunil-Chandra Narapity \\
\hline \multirow[t]{2}{*}{ Corea Enoka } & Kottahachchi Jananie & \\
\hline & Kudagammana Wasana & Tambyah Paul \\
\hline Dance David & & Tennegedara Asanka \\
\hline Danthanarayana Nayomi & Magana-Arachchi Dhammika & Tennekoon Imalka \\
\hline De Silva Chamari SH & Mallawaarachchi Harenda & \\
\hline De Silva Lalindi & Muthugala Rohitha & Wickramasinghe Darshana \\
\hline De Silva Nilhan & & Wijayaratne Gaya W. I. D \\
\hline De Zoysa Ishan & Nagahawatte Ajith & Wijesooriya Lakmini \\
\hline De Sllva Nelun & Noordeen Faseeha & \\
\hline \multirow{2}{*}{ Dissanayake Thushari } & & Yapa Lakmini \\
\hline & Panagoda Gehan & \\
\hline Fahim A.C.II & Pathirage Sujatha & \\
\hline Fernando Arsakulasooriya Lakkumar & Pathirana Sirira & \\
\hline \multirow[t]{2}{*}{ Fernando Neluka } & Perera Jennifer & \\
\hline & Perera Nelun & \\
\hline Gamage Chandika & Pieris Sriyal Malik & \\
\hline Goonesekera Chula & Piyasiri Bhagya Lasanthi & \\
\hline Gunasekara Chinthika & Premathilake Ishara & \\
\hline \multicolumn{3}{|l|}{ Gunatilake Nayana } \\
\hline \multirow[t]{2}{*}{ Gunawardena Sameera } & Ranganathan Shalini Sri & \\
\hline & Ratnatunga Champa & \\
\hline \multirow[t]{2}{*}{ Hapuarachchi Chaturika } & Refai Warda & \\
\hline & Rockwood Neesha & \\
\hline \multicolumn{3}{|l|}{ Janage Nadeeka } \\
\hline Jayasekera Primali & Samaranayake Nilakshi & \\
\hline
\end{tabular}

